\title{
Analysis of the role of the BMP7-Smad4-Id2 signaling pathway in SW480 colorectal carcinoma cells
}

\author{
QIANG SHI*, YUN-SHI ZHONG ${ }^{*}$, ZHONG REN, QUAN-LIN LI, \\ PING-HONG ZHOU, MEI-DONG XU and LI-QING YAO \\ Endoscopic Center, Zhongshan Hospital, Fudan University, Shanghai 200032, P.R. China \\ Received February 25, 2011; Accepted April 15, 2011
}

DOI: $10.3892 / \mathrm{mmr} .2011 .481$

\begin{abstract}
The bone morphogenetic proteins (BMPs) Smad4 and Id2 exert their effect on colorectal carcinoma via several uncharacterized mechanisms. In this study, we investigated whether the transcription factor Id2, which has been implicated in colorectal carcinoma proliferation and metastasis, is involved in BMP-7/Smad4 signaling, or whether it is regulated by BMP-7 via another mechanism in this cell type. A Smad4-cDNA vector was constructed and stably transfected into SW480 cells. Protein levels of Smad4 and Id2 were examined by Western blotting. Inhibitory effects on cellular proliferation activity were determined by the methyl thiazolyl tetrazolium (MTT) assay, and invasion and migration potential was detected using the in vitro Matrigel-coated invasion and migration assay. Levels of Smad4 protein were significantly increased in SW480 cells transfected with Smad4-cDNA, compared to those transfected with empty vector. Growth curve analysis revealed that live cell numbers were lower in the Smad4-expressing group than in the control group after $36 \mathrm{~h}$, and that BMP7 treatment caused an increase in live cell numbers in Smad4-expressing cells. Transwell chamber analysis revealed that migration/invasion activity was significantly suppressed when Smad4 was expressed. Finally, Smad4expressing cells treated with BMP7 expressed a higher level of Id2 protein than the controls. The results indicate that Smad4 expression may inhibit the growth and invasion of SW480 cells, and that BMP7 affects Id2 levels through Smad4. Therefore, BMP7-Smad4-Id2 signaling may play a significant role in the development of colorectal carcinoma.
\end{abstract}

\section{Introduction}

Smads are essential intracellular effectors of the transforming growth factor- $\beta$ (TGF- $\beta$ ) signaling pathway. Receptor-activated

Correspondence to: Professor Li-qing Yao, Endoscopic Center, Zhongshan Hospital, Fudan University, Shanghai 200032, P.R. China E-mail: shiqiangqy@126.com

\section{* Contributed equally}

Key words: colorectal carcinoma, SW480, cDNA, Smad4, BMP7, Id2
Smads bind Smad4 and translocate into the nucleus, where they cooperate with other transcription factors to activate or repress transcription. Smad4 is the core member of the Smad family. Bone morphogenetic proteins (BMPs) are ligands of the TGF- $\beta$ superfamily that initiate the signaling cascade, activating a heterodimeric complex of type I and II serine/threonine transmembrane receptor kinases (1). The BMP/Smad signaling pathway is crucial in tumor development. Id2 is a member of the Id helix-loop-helix (HLH) protein subfamily, which contains the HLH dimerization domain but lacks the basic DNA-binding domain, and is known to regulate proliferation and differentiation (2).

Preliminary observations suggest a correlation between BMP7 and Id2 expression in colorectal carcinoma $(3,4)$. Therefore, this study explored whether Id2 is a target of BMP7Smad4-mediated transcription or whether it is regulated by BMP7 via other mechanisms. A constructed vector carrying Smad4-cDNA was transfected into SW480 cells. The effects of Smad4 expression on cell proliferation and invasion were investigated, as was its interaction with BMP7. In this study, the relationship between BMP7, Smad4 and Id2 was analyzed for the first time. The results provide new evidence regarding the effects of the BMP7-Smad4-Id2 signaling pathway on colorectal cancer cells. Manipulating this pathway may represent a novel method for controlling the progression of colorectal carcinoma.

\section{Materials and methods}

Cell culture. The human colorectal carcinoma cell line SW480 was purchased from the China Centre for Type Culture Collection, Chinese Academy of Sciences. The cells were grown in Leibovitz L-15 medium (Genom) supplemented with $10 \%$ fetal bovine serum (FBS) (Biowest, South America) at $37^{\circ} \mathrm{C}$ in a humidified atmosphere of $5 \% \mathrm{CO}_{2}$. Cells were detached using Trypsin-EDTA and subcultured at $1.5 \times 10^{5}$ cells per well in 6-well tissue culture plates for transfection.

Plasmid construction. Promoterless luciferase vectors were purchased from Genechem Co. Ltd., Shanghai, China. The full-length coding sequence of DPC4/Smad4 (NM_005359) was derived from the pBK-DPC4 constitutive expression construct (5). The DPC4/Smad4 coding sequence was inserted into the XhoI/KpnI sites of the PEGFP-N1 vector. The construct was 

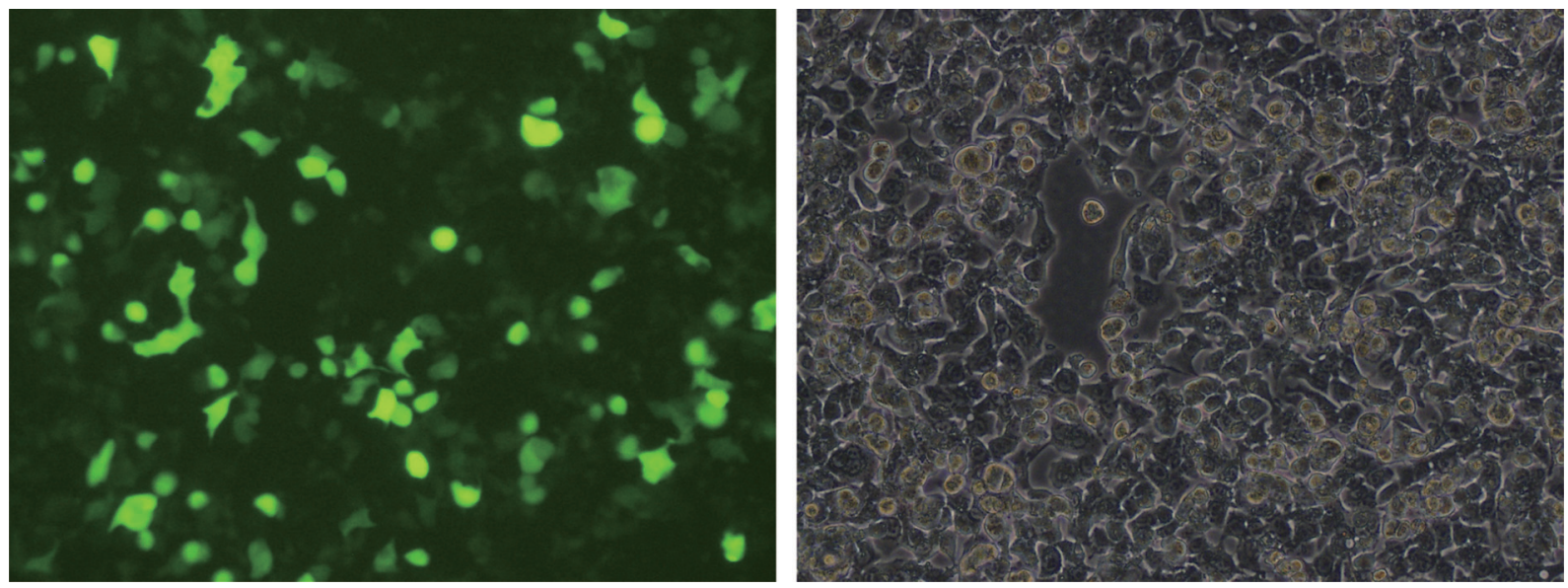

Figure 1. Expression of GFP in SW480 cells $48 \mathrm{~h}$ after transfection with Smad4-cDNA. The transfected cells display GFP expression (in green). The proportion of GFP-expressing cells is approximately 40\%. Magnification, x200.

confirmed by direct sequencing (SequiTherm Cycle Sequencing, Epicentre). All plasmids used for transfection were CsCl2purified, followed by proteinase $\mathrm{K}$ digestion, phenol extraction and precipitation.

Establishing Smad4 re-expression in SW480 cell lines. The targeting and control vector plasmid were transfected into SW480 cells using Lipofectamine 2000 (Invitrogen). The day before transfection, $1.5 \times 10^{5}$ cells per well were seeded into 6-well tissue culture plates containing antibiotic-free Leibovitz L-15 medium. On the day of transfection, Lipofectamine 2000 and plasmid were diluted in serum-free Opti-MEM medium (Invitrogen) at a ratio of 2.5:1, which had produced efficient transfection of SW480 cells in preliminary experiments. The mixture was separately transfected into the SW480 cells. The cells were cultured in medium supplemented with Geneticin (G418) (800 $\mu \mathrm{g} / \mathrm{ml}$, Invitrogen) for 2 weeks. Transfection efficiency was detected by directly testing the expression ratio of green fluorescent protein, and the G418-resistant clones were selected as stable transfectants. Stably transfected clones were selected and maintained in medium containing $400 \mu \mathrm{g} / \mathrm{ml}$ G418 for further experiments. SW480 cells stably transfected with the plasmid Smad4-cDNA were referred to as SW480Smad4-cDNA, and cells transfected with the empty plasmid were referred to as SW480-HK.

Western blot analysis. Cell samples were lysed in ice-cold lysis buffer (Beyotime, China) with $1 \%$ phenylmethylsulfonyl fluoride (PMSF) for $30 \mathrm{~min}$ and centrifuged at $10,000 \mathrm{x} \mathrm{g}$ for $20 \mathrm{~min}$ at $4^{\circ} \mathrm{C}$. The protein concentration of the resulting supernatant was determined by the bicinchoninic acid (BCA) protein assay kit (Beyotime, China). Proteins $(30 \mu \mathrm{g})$ were separated by $12 \%$ sodium dodecyl sulfate polyacrylamide gel (SDS-PAGE) electrophoresis and subsequently transferred to polyvinylidene fluoride (PVDF) membranes. The membranes were blocked with $5 \%$ non-fat dry milk in TBS/Tween 20 $(0.05 \%, \mathrm{v} / \mathrm{v})$ for $1 \mathrm{~h}$ at room temperature and incubated overnight at $4^{\circ} \mathrm{C}$ with primary antibodies directed against Smad4 (B8; Santa Cruz), Id2 (C-20; Santa Cruz) and $\beta$-actin (Santa Cruz). The blots were washed and incubated with secondary antibody [HRP-labeled goat anti-rabbit IgG and HRP-labeled goat anti-mouse $\operatorname{IgG}(\mathrm{H}+\mathrm{L})$ (Beyotime)], and developed with a chemiluminescent substrate (ECL Plus). Signals were detected using the AlphaImager EP Fluorescent and Visible Light Gel imaging system, and Photoshop CS was used for the analysis. Assays were performed in triplicate for each experiment, and each experiment was repeated three times.

MTT assay. For the measurement of cell proliferation rate, the collected cells stably transfected with SW480-Smad4-cDNA or SW480-HK were plated into 96-well plates at $5 \times 10^{3}$ cells/100 $\mu 1$ medium per well. After $24 \mathrm{~h}, \mathrm{BMP} 7$ (Peprotech Asia) at a concentration of $25 \mathrm{ng} / \mathrm{ml}$ was added to one of the SW480-Smad4-cDNA groups. Methyl thiazolyl tetrazolium (MTT) solution (10 $\mu$ l) (Amresco) was added to each well every $12 \mathrm{~h}$ from 12 to $48 \mathrm{~h}$, and the plates were incubated for $4 \mathrm{~h}$ at $37^{\circ} \mathrm{C}$. Subsequently, $150 \mu \mathrm{l}$ DMSO was added to dissolve the formazan crystals. Absorbance values (A) were measured at a wavelength of $490 \mathrm{~nm}$ using a microplate reader. Results were expressed as the mean \pm SEM. The assay was carried out in six wells, and each experiment was repeated three times.

In vitro Matrigel invasion assay. An in vitro Matrigel invasion assay was performed using a 24-well millicell insert (Corning) with polycarbonate filters (pore size, $8 \mu \mathrm{m}$ ). The upper side of the polycarbonate filter was coated with matrigel $(50 \mu \mathrm{g} /$ $\mathrm{ml}$, BD Biosciences). The chambers were incubated at $37^{\circ} \mathrm{C}$ with $5 \% \mathrm{CO}_{2}$ for $4 \mathrm{~h}$ to allow the matrix to form a continuous thin layer. The cells transfected with SW480-Smad4-cDNA or SW480-HK were then harvested, and $2 \times 10^{5}$ cells in $200 \mu \mathrm{l}$ $0.1 \%$ bovine serum albumin serum-free medium were placed in the upper chamber. The lower chamber was filled with $10 \%$ serum medium $(700 \mu \mathrm{l})$. Cells were cultured for $24 \mathrm{~h}$ at $37^{\circ} \mathrm{C}$ in $5 \% \mathrm{CO}_{2}$. Cells on the upper surface of the filter were removed using a cotton swab. Cells invading through the matrigel and filter to the lower surface were fixed with $4 \%$ neutral-buffered formalin for $30 \mathrm{~min}$ at room temperature and stained in Giemsa stain for $15 \mathrm{~min}$ at room temperature. The cell number in five fields (up, down, median, left and right, at a magnification of $\times 200$ ) of each chamber was counted, and the average value was calculated. Each invasion assay was performed in triplicate and repeated three times. 
A

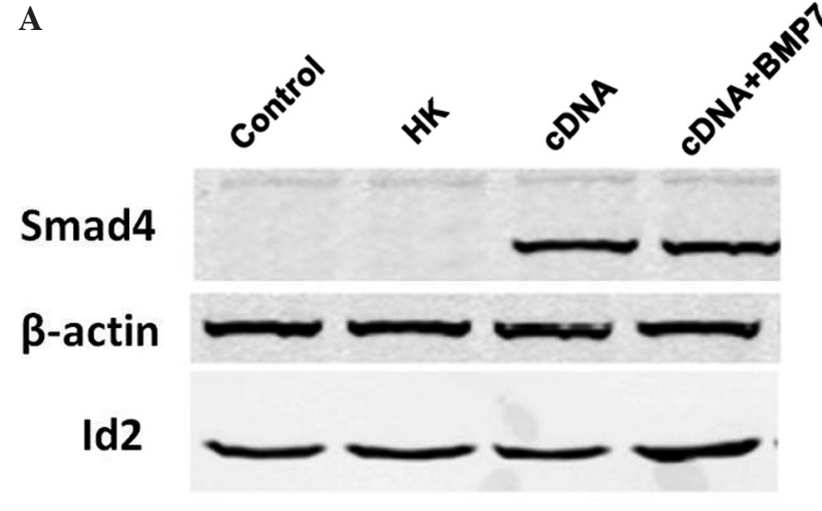

B

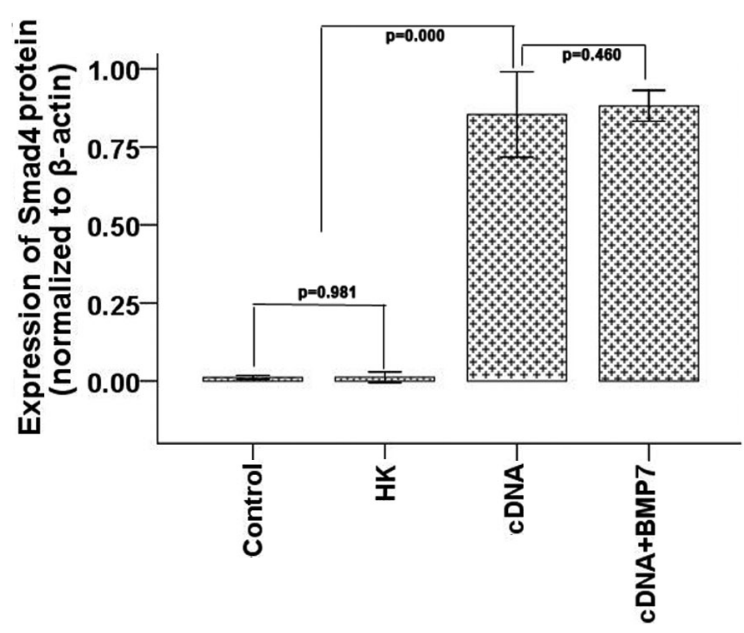

C

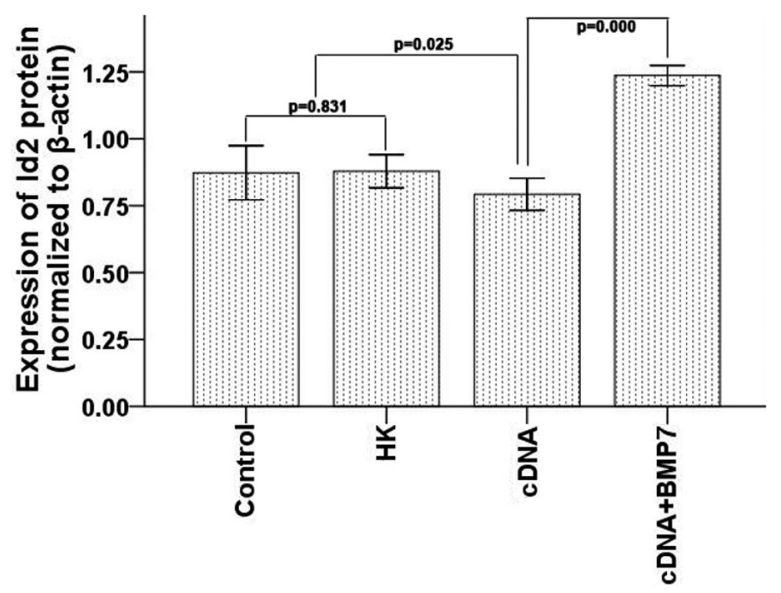

Figure 2. Smad4 and Id2 protein levels in SW480 cells. (A) Smad4 protein was expressed by transfecting cells with Smad4-cDNA. Id2 protein levels in Smad4-expressing cells were significantly reduced compared to control cells or cells transfected with HK. Id2 levels were increased in Smad4-expressing cells treated with BMP7, compared to untreated cells. $\beta$-actin was used as a loading control. (B and C) The graphs show the signal intensity of Smad4 and Id 2 protein levels as detected by Western blotting, quantified with ImageJ software.

In vitro cell migration assay. A cell migration assay was used to measure cell migration through an $8-\mu \mathrm{m}$ pore size membrane in a 24-well millicell insert. The lower chamber was filled with $10 \%$ serum medium $(700 \mu \mathrm{l})$. Cells $\left(2 \times 10^{5}\right)$ in $200 \mu \mathrm{l}$ medium

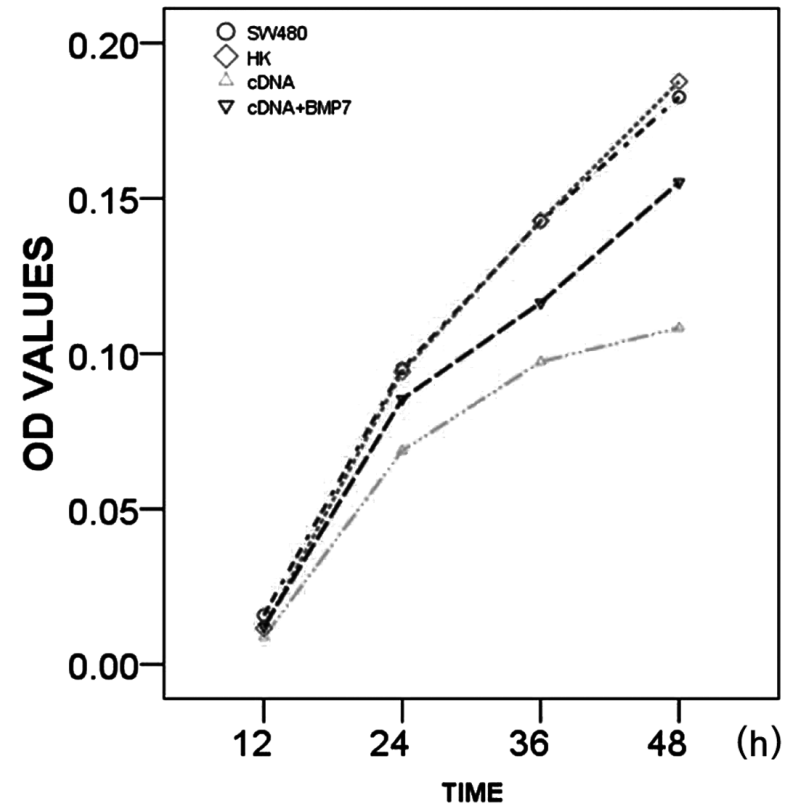

Figure 3. Effect of Smad4 and BMP7 on cell growth . Cell proliferation was measured by light absorption at a wavelength of $490 \mathrm{~nm}$. At $36 \mathrm{~h}$, a significant inhibition of cell growth was observed in Smad4-expressing cells. Non-significant differences were observed between untransfected cells and cells transfected with HK. BMP7 affected proliferation at $48 \mathrm{~h}$.

supplemented with $10 \%$ FBS were placed in the upper chamber. After $24 \mathrm{~h}$ of incubation, the number of migrated cells (lower side of the membrane) was counted as described above.

Statistical analysis. Statistical analyses were performed using SPSS statistical software (SPSS Inc, Chicago, IL, USA). Data were expressed as the mean \pm SEM. Statistical significance of differences between groups was performed by one-way analysis of variance (ANOVA). $\mathrm{P}<0.05$ was considered statistically significant. The SNK test was used to analyze the difference between two groups.

\section{Results}

Transfection of SW480 cells with Smad4. Through sequence analysis, a vector containing Smad4-cDNA was verified to be correctly constructed. There were no significant differences in terms of transfection conditions or efficiency between the experimental and control groups (Fig. 1). A comparison of fluorescence and bright field images of transfected cells revealed that the transfection efficiency for Smad4-cDNA and the empty vector (HK) was $\sim 40 \%$. Cells with stable integration of the cDNA were selected by treatment with G418 for 14 days.

Smad4 expression changes the level of Id 2 protein in SW480 cells. After transfection of Smad4-cDNA or HK into SW480 cells, the level of Smad4 protein was analyzed by Western blotting. The signal intensity of Smad4 protein was significantly higher in cells transfected with Smad4-cDNA than in untransfected cells or cells transfected with HK (F=689.839, $\mathrm{P}<0.05$ ) (Fig. 2). In SW480 cells with increased Smad4, the level of Id 2 protein was reduced $(\mathrm{F}=7.232, \mathrm{P}<0.05)$. The signal 
A
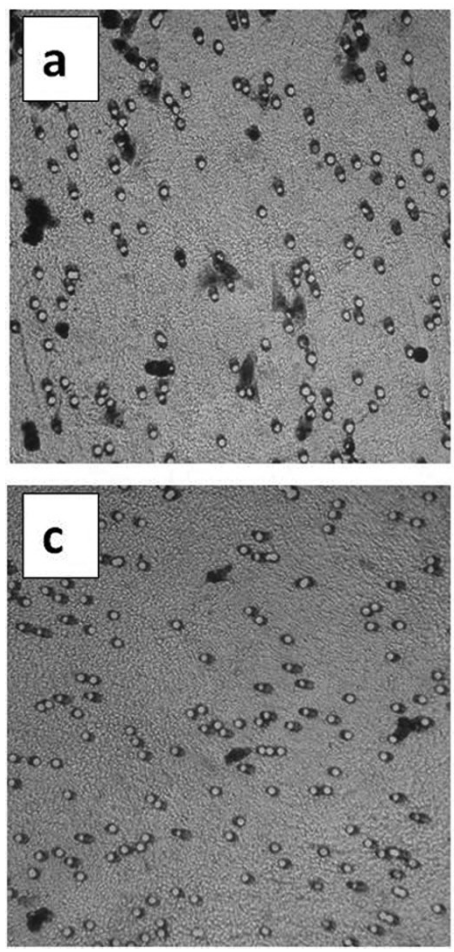
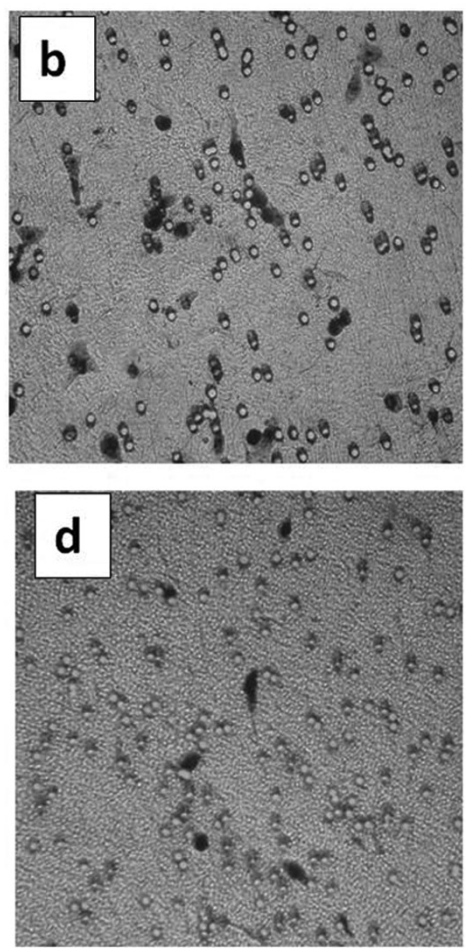
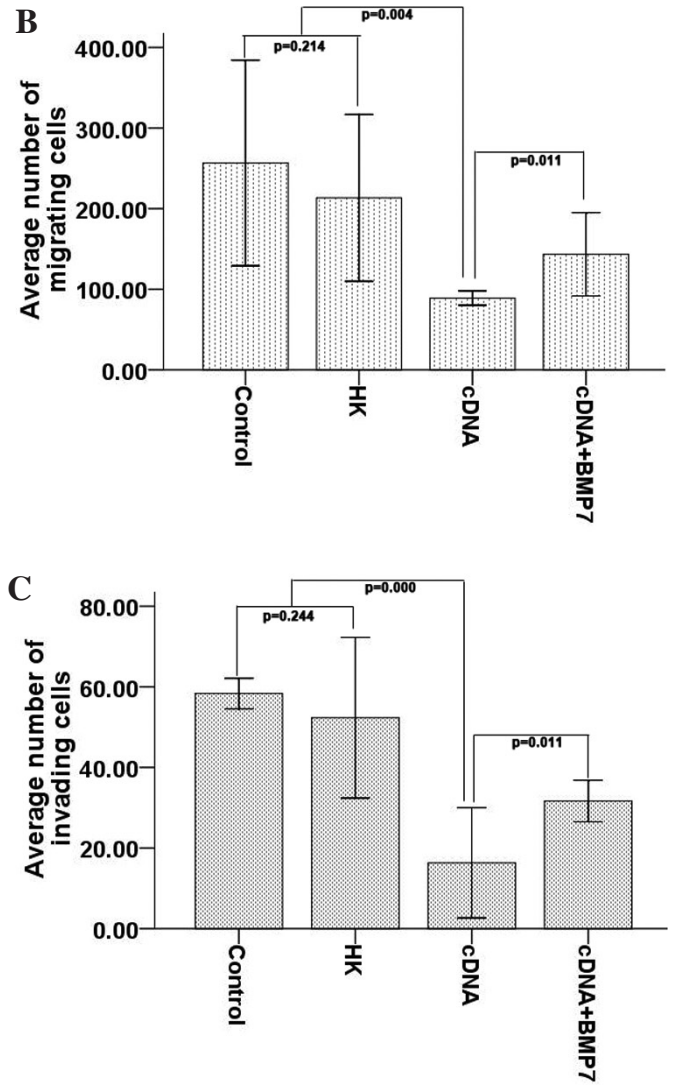

Figure 4. Inhibitory effect of Smad4 on invasion and migration in SW480 cells. (A) The number of invading cells was determined by counting cells stained with Giemsa on the lower side of the membrane (a, SW480 control cells; b, HK-transfected cells; c, Smad4 cDNA-transfected cells; d, Smad4-cDNA+BMP7 treatment). (B and C) The graphs compare the number of transmembrane cells in the invasion and migration experiments. Data represent the mean \pm SEM of three independent experiments.

intensity of Id 2 protein for Smad4-expressing cells treated with BMP7 was significantly higher than that of untreated cells $(\mathrm{F}=721.601, \mathrm{P}<0.05)$ (Fig. 2).

Effects of Smad4 expression on cell proliferation. Cell proliferation is essential for tumorigenesis. Collected cells were seeded onto 96-well microplates and proliferation rates were determined by continuous MTT assays, performed every $12 \mathrm{~h}$. Growth curves were plotted using the dynamic OD values obtained in the MTT assays. The differences in cell growth rate between SW480 cells transfected with Smad4-cDNA and the other three groups were not statistically significant in the first $24 \mathrm{~h}$. However, after $36 \mathrm{~h}$, significant differences $(\mathrm{F}=4.377$, $\mathrm{P}=0.037$ ) were observed between SW480 cells transfected with Smad4-cDNA and those transfected with HK (Fig. 3). No significant difference was found between untransfected cells and cells transfected with HK. A significant difference was observed after $48 \mathrm{~h}$ between Smad4-expressing cells treated with $25 \mathrm{ng} / \mathrm{ml}$ BMP7 and cells not exposed to BMP7 ( $F=23.544, \mathrm{P}=0.001)$ (Fig. 3). These results show that the expression of Smad4 in SW480 cells reduces the cell proliferation rate in vitro, and that BMP7 treatment partially inhibits this effect.

Effect of Smad4 expression on cell invasion and migration in vitro. After incubation for $24 \mathrm{~h}$, control SW480 cells showed a stronger invasion potential than Smad4-transfected cells $(\mathrm{F}=47.876, \mathrm{P}<0.001)$ (Fig. 4). There was no statistically signif- icant difference between untransfected and HK-transfected cells. The transmembrane invasion capacity of SW480 cells treated with BMP7 differed significantly compared to the controls ( $\mathrm{F}=20.346, \mathrm{P}=0.011)$. Moreover, Smad4-expressing SW480 cells displayed significantly lower transmembrane migration activity compared to the HK-transfected cells or untransfected controls $(\mathrm{F}=15.566, \mathrm{P}=0.004)$. The transmembrane migration activity of SW480 cells treated with BMP7 was significantly affected $(\mathrm{F}=19.842, \mathrm{P}=0.011)$ (Fig. 4). These findings suggest that increased Smad4 levels enhance invasion and migration in SW480 cells. Furthermore, they indicate that BMP7 has a significant effect on Smad4-expressing cells.

\section{Discussion}

Recently, the relationship between transforming growth factor- $\beta$ (TGF- $\beta$ ) and cancer development has been examined from various perspectives. As a multifunctional growth factor, TGF- $\beta$ has both tumor suppressor and oncogenic activities, depending on the stage of carcinogenesis and the responsiveness of the tumor cells $(6,7)$. BMPs, which are members of the transforming growth factor- $\beta$ (TGF- $\beta$ ) superfamily, were first identified by their ability to induce ectopic bone formation (8). Subsequently, BMPs have been shown to play significant roles in axis determination, at stages as early as gastrulation, as well as during the development of numerous organs $(9,10)$. BMPs initiate the signaling cascade by binding as a ligand, activating a complex of heterodimeric type I and II serine/ 
threonine transmembrane receptor kinases (1). The activated BMP type I receptor phosphorylates Smad1 and Smad5, resulting in their dissociation from the receptor complex. Phosphorylated Smad1 and Smad5 then form heterodimeric complexes with Smad4, translocate into the nucleus and activate the transcription of target genes (11-13). The BMP/Smad signaling pathway is also negatively regulated by structurally and functionally divergent members of the inhibitory Smad subfamily: Smad6 and Smad7. The role of BMPs in colorectal carcinoma progression has been well-characterized. However, the mechanisms involved in their activation and/or regulation, which specifically contribute to tumor progression and metastasis, have not been fully elucidated.

Smad family members, as effectors of the TGF- $\beta$ signaling pathway, play a key role in TGF- $\beta$ signal transduction. Smads have been classified into three subtypes according to their structures and function: receptor-activated Smads (R-Smads), the common mediator Smad (Co-Smad), Smad4, and inhibitory Smads (I-Smads) (14,15). Smad proteins have a conserved N-terminal MH1 domain, a conserved C-terminal MH2 domain, and a non-conserved linker region. The MH1 domain is highly conserved among R-Smads and Co-Smad. Functionally, this domain is implicated in nuclear import and transcription by binding to DNA and interacting with nuclear proteins, such as the vitamin D receptor (VDR), the transcription factor Jun, transcription factor E3 (TFE3) and activating transcription factor 2 (ATF2) (16). The MH2 domain is highly conserved among all Smads.

Smad4 is an essential tumor suppressor gene. When Smad4 is mutated or deleted, the TGF- $\beta$ signal transduction network cannot be activated, and the pathway loses its effect on tumor development. In the present study, Smad4 was expressed in the colon cancer cell line SW480 by transfection with Smad4-cDNA. To investigate the contribution of BMP7 and Smad4 to colon cancer cell behavior, Smad4-expressing cells were treated with BMP7. These experiments revealed that when Smad4 is expressed in colorectal cancer cells, cell proliferation, migration and invasion are decreased, indicating that Smad4 is a tumor suppressor gene. This finding is corroborated by the results of previous studies. On the other hand, we found that when Smad4-expressing cells are exposed to BMP7, cell proliferation and migration are enhanced. In a previous study, we showed that BMP7 acts on the colorectal cancer cell line HCT116. When Smad4 was inhibited in this cell line by Smad4-shRNA, the proliferation, migration and invasiveness of the cells was unchanged. This demonstrates that BMP7 functions via Smad4 in colorectal cancer cells. This study goes further, by taking into account the role of Id 2 in colorectal cancer, as corroborated by a recent study examining the relationship between the expression of BMP7 and Id 2 in colon cancer (3). In the present study, we investigated whether Id 2 is downstream of BMP7/Smad4 signaling, or whether BMP7 regulates this gene by another mechanism. Western blot experiments demonstrated that the expression of Id 2 increases when cells are exposed to BMP7. This confirms our hypothesis that the BMP7-Smad4-Id2 pathway affects colon cancer cells, and may play a role in the development of colon cancer.

To our knowledge, this is the first study to reveal the relationship between BMP7, Smad4 and Id 2 in colorectal cells.
The study provides significant insights into tumorigenesis, and may aid in the identification of effective new targets for gene therapy.

\section{Acknowledgements}

This study was supported by grants from the Natural Scientific Foundation of Shanghai Province (no. 09JC1403300) and the Youth Foundation of Shanghai Health Bureau (no. 2007Y38)

\section{References}

1. Chen D, Zhao M and Mundy GR: Bone morphogenetic proteins. Growth Factors 22: 233-241, 2004

2. Rockman SP, Currie SA, Ciavarella M, et al: Id2 is a target of the beta-catenin/T cell factor pathway in colon carcinoma. J Biol Chem 276: 45113-45119, 2001.

3. Fritah A, Saucier C, De Wever O, et al: Role of WISP-2/CCN5 in the maintenance of a differentiated and noninvasive phenotype in human breast cancer cells. Mol Cell Biol 28: 1114-1123, 2008.

4. Kowanetz M, Valcourt U, Bergstrom R, Heldin $\mathrm{CH}$ and Moustakas A: Id 2 and Id 3 define the potency of cell proliferation and differentiation responses to transforming growth factor beta and bone morphogenetic protein. Mol Cell Biol 24: 4241-4254, 2004.

5. Schwarte-Waldhoff I, Klein S, Blass-Kampmann S, et al: DPC4/ SMAD4 mediated tumor suppression of colon carcinoma cells is associated with reduced urokinase expression. Oncogene 18: 3152-3158, 1999.

6. Wakefield LM and Roberts AB: TGF-beta signaling: positive and negative effects on tumorigenesis. Curr Opin Genet Dev 12: 22-29, 2002.

7. Seoane J, Le HV, Shen L, Anderson SA and Massague J: Integration of Smad and forkhead pathways in the control of neuroepithelial and glioblastoma cell proliferation. Cell 117: 211-223, 2004.

8. Wozney JM, Rosen V, Celeste AJ, et al: Novel regulators of bone formation: molecular clones and activities. Science 242: 1528-1534, 1988.

9. Hogan BL: Bone morphogenetic proteins: multifunctional regulators of vertebrate development. Genes Dev 10: 1580-1594, 1996.

10. Thompson DL, Gerlach-Bank LM, Barald KF and Koenig RJ: Retinoic acid repression of bone morphogenetic protein 4 in inner ear development. Mol Cell Biol 23: 2277-2286, 2003.

11. Derynck R, Zhang Y and Feng XH: Smads: transcriptional activators of TGF-beta responses. Cell 95: 737-740, 1998.

12. Attisano L and Wrana JL: Smads as transcriptional co-modulators. Curr Opin Cell Biol 12: 235-243, 2000.

13. Massague $\mathrm{J}$ and Wotton D: Transcriptional control by the TGF-beta/Smad signaling system. EMBO J 19: 1745-1754, 2000.

14. Wrana JL and Attisano L: The Smad pathway. Cytokine Growth Factor Rev 11: 5-13, 2000.

15. Chen W, Fu X and Sheng Z: Review of current progress in the structure and function of Smad proteins. Chin Med J (Engl) 115: 446-450, 2002.

16. Moustakas A, Souchelnytskyi S and Heldin $\mathrm{CH}$ : Smad regulation in TGF-beta signal transduction. J Cell Sci 114: 4359-4369, 2001. 